\title{
07 | Experimentación radical italiana en torno al night-club Warhol-McLuhan-Price y la arquitectura eléctrica de los años 60
} Marcos Parga

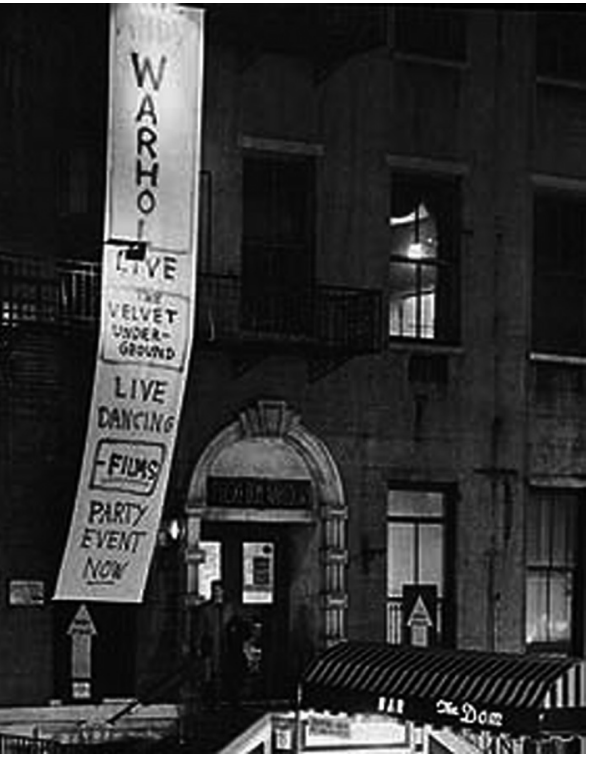

[1]

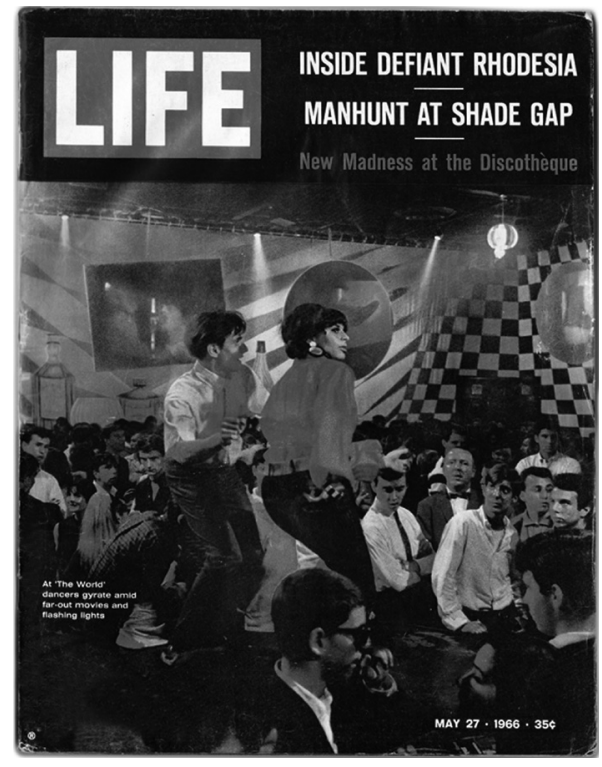

[2]

En enero de 1966 Andy Warhol reúne a Nico y a la Velvet Underground de Lou Reed junto con algunos performers y varios personajes asiduos de la Factory y, utilizando como telón de fondo imágenes de sus últimas películas, los pone a actuar en el club DOM de Saint Mark's Place [1], en el East Village neoyorkino, reconvertido un año más tarde en el famoso Electric Circus ${ }^{1}$.

El experimento supone la puesta en escena del primero de una serie de eventos multimedia diseñados por Warhol que, influenciado por la inauguración casi simultánea también en Nueva York del club The Cheetah ${ }^{2}$ (El Guepardo) y las colaboraciones en forma de proyecciones que Jonas Mekas había incorporado durante varios conciertos en The Cinematheque, tendrán lugar periódicamente en diferentes locales del país durante los dos años posteriores, primero bajo el nombre de "Andy Warhol, Up-Tight" y más tarde definitivamente como Exploding Plastic Inevitable (EPI) ${ }^{3}$.

Las sesiones consistían en un impactante espectáculo multidisciplinar y colectivo que transformaba el espacio en una nueva forma de "entorno" mediante la sobrecarga sensorial y un alto grado de estimulación combinados con bajos niveles de resolución visual y auditiva, que lo convertían en el escenario perfecto para todo tipo de performances sincronizadas que incluian improvisaciones musicales, proyecciones de películas, screen tests (los "retratos" audiovisuales que Warhol hacía a sus superestrellas y a otras personas que circulaban por la Factory), fotografías, luces estroboscópicas, danza, e incluso rodajes cinematográficos. A modo de declaración de intenciones, esta primera experiencia en el club DOM combinaba las películas de Warhol, las luces de Danny Williams, la música de la Velvet Underground, la voz de Nico, la danza de Gerard Malanga y los bailes de Edie Sedgwick, las diapositivas y proyecciones de Paul Morrissey y Warhol, las fotografías de Billy Linich y de Nat Finkelstein, el rodaje cinematográfico de Barbara Rubin, "y el público haciendo de público", tal y como rezaba la publicidad del evento.

Inmediatamente las E.P.I. del Electric Circus se incorporan al panorama del floreciente ocio nocturno [2] gracias a una oferta irresistible y perfectamente programada escenificada en un interior envolvente -diseñado por el arquitecto Charles Forberg a base de membranas de nailon curvado- sobre el que se proyectaban las imágenes que junto a la música y la puesta en escena generaban el pretendido -y rentable- caos multimedia [3]. El local se convierte así en uno de los primeros ejemplos de interacción electrónica (luz-sonido-espacio) comercialmente transformada en acontecimiento social consumido masivamente por aquellos jóvenes que, en plena era psicodélica y sacudidos por el discurso hippie-campestre, abrazaban la contracultura desde ámbitos underground en busca de nuevas experiencias sugeridas a través de incisivas

Resumen pág 56 | Bibliografía pág 61

Marcos Parga (Lugo, 1971) es arquitecto por la ETSA de Madrid, donde, desde 2008, es Profesor Asociado de Proyectos Arquitectónicos, miembro del Grupo de Investigación "Cultura del Hábitat" y editor de la revista académica 115Días.

En 2014, tras 15 años como director de PO2 Arquitectos, funda el estudio MAPAA (www.mapaa.es) con la intención de explorar nuevas vías de conexión productiva entre investigación, docencia y la imprescindible experiencia constructiva.

En la actualidad desarrolla su Tesis Doctoral en el Departamento de Proyectos Arquitectónicos de la ETSAM en torno a la Arquitectura Radical de los años 60 bajo el título "La opción radical. Viaje a través de Superstudio".

\section{Palabras clave}

Andy Warhol, E.P.I., night-club, contracultura, entornos amplificados, Marshall McLuhan, Cedric Price, Leonardo Savioli, Arquitectura Radical, Piper Club Phenomenon

1 En 1967, Jerry Brand y Stanton J. Freeman se hacen cargo del club DOM y lo transforman en el Electric Circus, nombre que hace referencia a su intención de convertir el local en un "circo electrónico" capaz de albergar todo tipo de espectáculos creando lo que Brandt pronto denominó como Ultra-Media, una brutal y rentable fusión de luces, música y espectáculos en directo: el lugar perfecto para escenificar los "experimentos" de Warhol.

2 A finales de mayo de 1966 se abre en Nueva York el club The Cheetah, espacio de aspecto cavernoso y grandes dimensiones cuya visita pronto es descrita publicitariamente como "a psychedelic experience" gracias a la incorporación de un sofisticado equipamiento que permitía reproducir impactantes y envolventes efectos de luz y sonido. Jonas Mekas, cineasta experimental y noctámbulo habitual, describía su experiencia en el local apuntando que "The Cheetah provides the most curious use of the intermedia" "The Cheetah supone el uso más curioso de los medios audiovisuales"] y resaltaba su "popularidad" frente a la oferta experimental del club DOM: "Whereas the Dom shows are restricted (or became restricted) to the In-circle, Cheetah was designed for the masses". ["Mientras los espectáculos del DOM se restringen (o se convierten en restringidos) a un circulo intimo, el Cheetah estaba diseñado para las masas"]. WATSON, Steven. Factory Made: Warhol and the Sixties. New York: Pantheon Books, 2003. 3 E.P.I., o Exploding Plastic Inevitable, fue un happening multisensorial que durante año y medio reunió periódicamente a colaboradores de Warhol en la Factory con la Velvet Underground. Aunque el acontecimiento de las EPI comenzó siendo muy pequeño, su onda expansiva se puede intuir en la asimilación y posterior generalización 


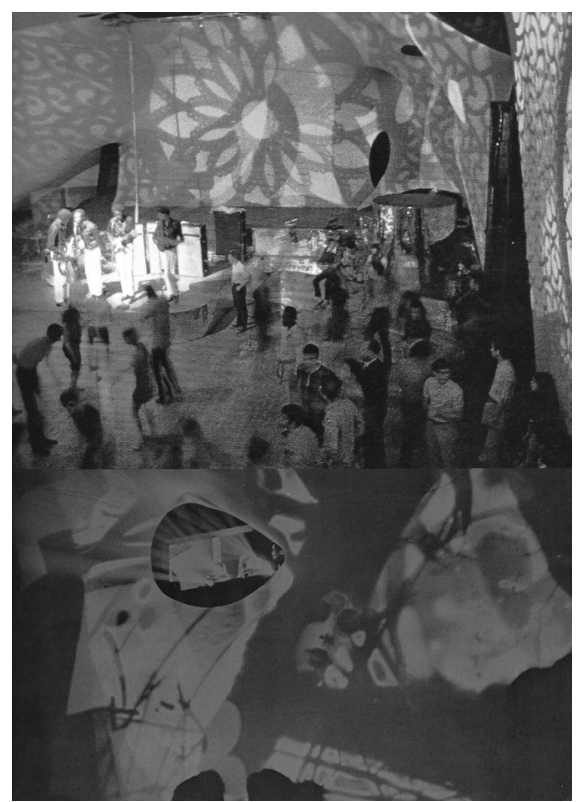

[3]

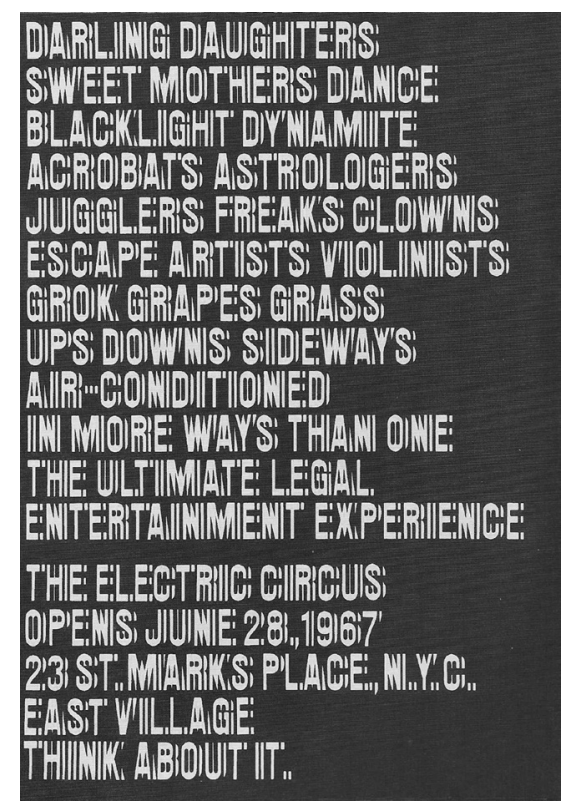

[4]
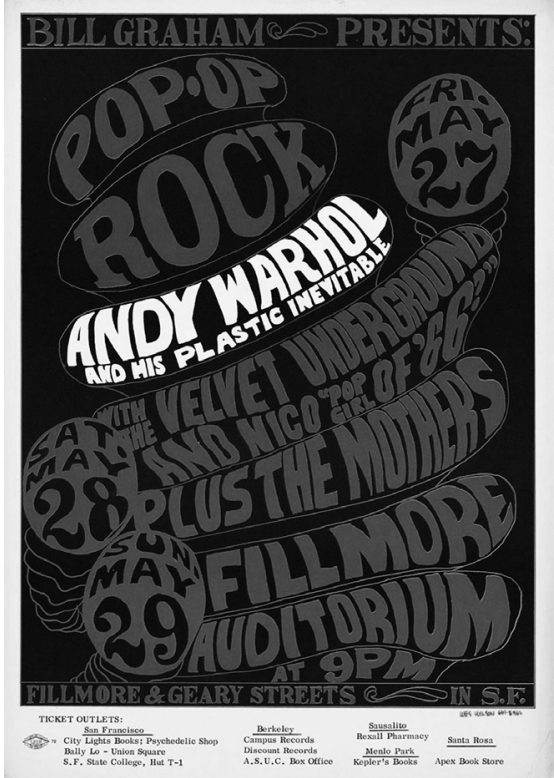

[5] del concepto multimedia dentro de un espectáculo de rock y en el comienzo de una alianza no siempre bien entendida entre arte $y$ rock. Paul Morrissey, uno de los colaboradores de Andy Warhol, cuenta que "El término 'Exploding Plastic Inevitable' se nos ocurrió un día que estábamos con Gerard y Barbara Rubin. (...) Había un texto anfetamínico e incoherente de Bob (Dylan). Lo miré por encima y vi tres palabras: algo era exploding, algo era plastic, algo era inevitable". Para más información sobre las EPI ver JOSEPH, Branden W. My mind split open: Andy Warhol's Exploding Plastic Inevitable. Grey Room 8, Verano 2002. pp. 80-107.

4 "There are moments, at the Dom (...) when I feel I am witnessing the beginnings of new religions, that I find myself in religious, mystical environments where the ceremonials and music and body movements and the symbolism of lights and colors are being discovered and explored. The very people who come to these shows have all something of a religious bond among them. Something is happening and is happening fast-and it has something to do with light, it has everything to do with light...". ["Hay momentos en el DOM en los que siento que estoy asistiendo a los comienzos de una nueva religión, que me encuentro en un entorno religioso y místico donde ceremonias, música y movimientos corporales, $\mathrm{y}$ el simbolismo de luces $\mathrm{y}$ colores están siendo descubiertos y explotados. Las mismas personas que acuden a estos espectáculos tienen todos algo de un vínculo religioso entre ellos. Algo está pasando y está pasando muy rápido -y es algo que tiene que ver con la luz, tiene todo que ver con la luz..."]. MEKAS, Jonas. Movie Journal 244 Junio 1966.

5 "It is a place where Marshal McLuhan meets Sigmun Freud". James Lapsley citado en GORDON, Alastair. Spaced Out: Radical Environments of the Psychedelic Sixties. New York: Rizzoli, 2008, p.57. Texto origina escrito tras su visita al Electric Circus en 1968, LAPSLEY, James. A Psycho-theological appraisal. Theology Today, enero 1969, pp. 435-445.

[6]

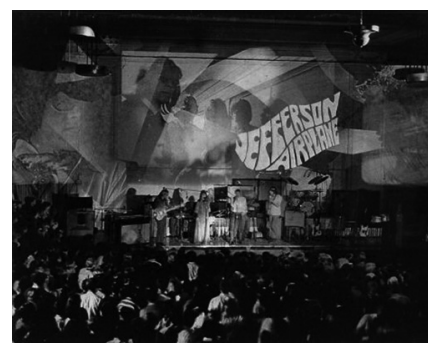

campañas de publicidad que prometían "in more ways than one the ultimate legal entertaiment experience"4 ["en más de un sentido, la experiencia definitiva de entretenimiento legal"[4]

En pocos meses el "Circo Eléctrico" de Nueva York o el Fillmore Auditorium de San Francisco $[5,6]$ se convierten en improvisados escenarios del lado más salvaje y creativo de la cultura de club de los años 60, alternando las EPI con espectáculos de luz, conciertos de música, atracciones circenses y teatro experimental para alimentar el conocido mantra "play games, dress as you like, dance, sit, think, tune in and turn on" ["juega, vístete como quieras, baila, siéntate, piensa, conéctate o desconéctate"] con el que pretendían trascender la discoteca tradicional y acercarse a lo ritual, convirtiéndose, como apuntaba el teólogo James Lapsley, en aquellos lugares en los que Marshal McLuhan y Sigmund Freud se encontraban, una Gesamtkunstwerk [obra de arte total] abierta al público 5 .

La atinada frase de Lapsley tiene que ver con el inesperado interés que las primeras EPI despiertan en un lúcido McLuhan, por aquellos años empeñado en avanzar las implicaciones de una nueva sociedad de la información ${ }^{6}$ mediante el análisis del espacio visual de la misma forma que Freud había hecho con el sexo -es decir, tratando de revelar su omnipresencia en la estructuración de las relaciones humanas-, quien pronto detecta en estos eventos su potencial impacto cultural y los utiliza como modelos de lo que en su libro de 1967, The Medium is the Massage: an Inventory of effects, definiria como "allatonceness" (all-at-onceness, todo al mismo tiempo, simultaneidad) para describir la "planeidad" del incipiente mundo digital presidido por la "televisión" que proclamaba la desaparición de las coordenadas espacio-temporales. Metafóricamente, McLuhan consideraba a las EPI más "televisión" que la propia "televisión", ya que llevaban al extremo la participación y lo inmersivo al hacer realidad los anhelos de fusión colectiva de los nuevos clubbers ${ }^{7}$, convertidos en parte activa y emancipada del proceso de construcción del evento para llenar el espacio anodino del club con una nueva forma de entorno hiper-estimulado [7] que borraba la legibilidad del marco arquitectónico hasta convertirlo en un mero contenedor activado donde el show lumínico era el único espacio posible ${ }^{8}$.

[1] Exterior del club The DOM en St. Mark's Place, East Village. NYC (1966). Fuente: warholstars.org

[2] Portada de la revista LIFE (mayo,1966) en la que se hace eco de la "locura discotequera" surgida ese año. "New Madness at the Discotheque", New York, 1966 (Life, September 9, 1966). En GORDON, Alastair. Spaced Out. Radical environments of the psychedelic sixties. New York: Rizzoli, 2008, p.48

[3] "Ultramedia", espectáculo de Tony Martin para el Electric Circus (1967). Interior envolvente diseñado por Charles Forberg. Arriba CPhoto Anne Simpkin, 1967. Abajo CPhoto Malcolm Varon, 1967. Fuente: GORDON, Alastair. Spaced Out. Radical environments of the psychedelic sixties. New York: Rizzoli, 2008, p.53.

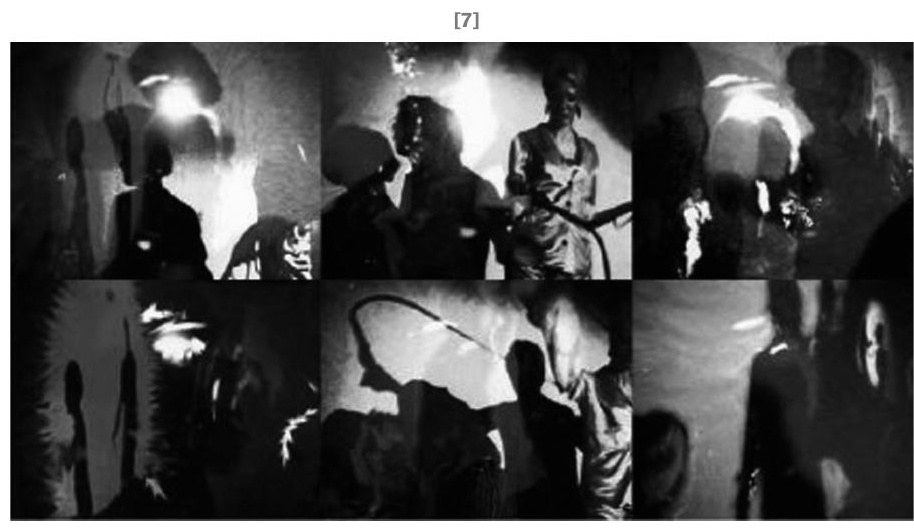


${ }^{13}$ En 1971 sale a la venta el mítico número 2/3 de la revista IN editada en Milán por Ugo La Pietra y Pierpaolo Saporito para dar visibilidad a las grandes voces del debate internacional del momento. Germano Celant colabora para este número con el artículo "Senza titolo" en el que por primera vez se utiliza el término "arquitectura radical" para designar el trabajo de los grupos contraculturales de la segunda mitad de los años 60.

${ }^{14}$ Para más información sobre el nacimiento y expansión de la "era pop" ver el artículo de FOSTER, Hal. Image building. Radical City 01, Archphoto 2.0, 2011.

${ }^{15}$ GINSBORG, Paul. A history of contemporary Italy: society and politics, 1943-1988. Londres: Penguin UK, 1990. p. 214 .

${ }^{16}$ El movimiento que aglutina las luchas de la clase obrera en Italia se denomina "operaismo". Es de carácter marxista, heterodoxo y anti-autoritario y detrás de él se encuentran pensadores y filósofos como Antonio Negri o Mario Tronti.

${ }^{17}$ Los escritos de Mario Tronti son especialmente decisivos en la actitud inicial de los jóvenes arquitectos italianos descontentos con el sistema en el que les ha tocado trabajar $y$, en particular, las "estrategias de rechazo" defendidas por el activista politico son el germen de las futuras posiciones de "silencio absoluto" o retirada de la actividad convencional practicada por grupos como Archizoom y Superstudio.

${ }^{18}$ Ver WOLF, Amit. "Discorsi per Immagini: Of Political and Architectural Experimentation". eScolarship, University of California. 2012.

19 Para profundizar en las relaciones entre arquitectura y política en este periodo ver AURELI, Pier Vittorio. The project of autonomy. Politics and architecture within and against capitalism. Princeton Architectural Press, 2008.

${ }^{20}$ En 1964 se produce la toma simbólica del despacho del Rector de la Facultad de Arquitectura de Florencia por parte de los estudiantes, acto en el que estaban involucrados varios de los futuros protagonistas de la arquitectura radical florentina, y que supuso un pequeño éxito para el movimiento estudianti.
[7] Diferentes escenas de la EPI "Taste the whip" en el Electric Circus (1967) CPhoto Ronald Nameth, 1967.

[8] Interior del club VOOM VOMM en Saint Tropez. Proyecto de Paul Bertrand con instalación de Nicolas Schöffer (1965). CPhoto Daniel Frasnay, 1956

[9] CYSP1 de Nicolas Schöffer (1956), primera escultura "espaciodinámica" Fuente: www. olats.org/schoffer/archives/voomvoom.html

[10] Entrada del Piper Club de Roma (1965). (C) Photo Federico Garolla, 1965

[11] Patty Pravo representando "The Pied Piper" en el Piper Club de Roma (1965). (c) Photo Fot. Ancillotti, 1964.

[12] Caleidoscopio, de V.Gregotti, G.Stoppino, L.Meneguetti y P.Brivio. Instalación multimedia en la XIII Triennale di Milano (1964), cuya "Sezione introduttiva a carattere internazionale"
estaba comisariada por Umberto Eco y Vittorio Gregotti y dedicada al "Tempo libero". Fuente: www.derossiassociati.it

[13] Interior del Piper Pluriclub de Turin (1966) Fuente: architetturaradicale.blogspot.co.uk

[14] Interior del Piper Pluriclub de Turin (1966) Fuente: architetturaradicale.blogspot.co.uk

[15] Proyección de la cúpula del Museo Guggenheim de Nueva York en el apartamento de C.Ray Smith, ejecutado por D.Gersztoff, J.Nuckolls y W.Warfel. CPhoto Louis Reens,
1967. 1967. internacional, convirtiéndose en lugar de reunión de los amantes de la "dolce vita" local [11] y, a la vez, en el desencadenante que da paso a un intenso periodo en el que paradójicamente la discoteca se revela como el centro del debate arquitectónico en un país -Italia- sumido en un agitado clima socio-político que dará lugar a lo que años más tarde Germano Celant definiría como "Arquitectura Radical"13.

Conviene recordar aquí que diez años antes aquella Europa, donde ahora empezaban a florecer los nuevos locales de ocio nocturno, había asistido atónita a la expansión de la influencia estadounidense en términos económicos -por obra y gracia del Plan Marshall- en forma de un nuevo "imperialismo" que propiciaría la importación de su invasiva cultura de masas. El consiguiente auge de la cultura $p o p^{14}$ desencadena una serie de reacciones paralelas que se podrían enmarcar en un amplio movimiento vinculado a la cada vez más incuestionable necesidad de revisión de los fundamentos teóricos heredados, afectando de manera trascendental a todas las disciplinas en un momento de agitación política y social motivada por la demanda de cambios que culminará con la puesta en escena de Mayo del 68 francés.

Italia no es ajena a este fenómeno. Desde mediados de los años 50 hasta principios de los 60 el país experimenta un crecimiento económico sin precedentes basado en un nuevo sistema industrial ligado por un lado a una creciente demanda interna y por otro a la exportación de bienes de consumo demandados por los países industrializados de su entorno ${ }^{15}$. Bautizado como "Milagro económico" este periodo de feroz incremento de la producción y la exportación también conlleva una intensa transformación social motivada por los desequilibrios que este crecimiento produce, ya que supone el paso de una estructura eminentemente rural a otra fundamentalmente industrial donde las grandes migraciones sur-norte precipitan la aparición de grandes aglomeraciones urbanas habitadas principalmente por trabajadores no cualificados procedentes del campo.

Instigados por las nuevas desigualdades sociales y geográficas, los movimientos obreros ${ }^{16}$ adquieren un protagonismo social sin precedentes, basando su actividad reivindicativa en teorías marxistas ahora redescubiertas por revistas como los Quaderni Rossi (1961-65) de Mario Tronti ${ }^{17}$ o la escindida Classe Operaria [Clase Obrera] (1963-66), cuyo grupo editorial estaba íntimamente ligado a la "Lega Architetti Studenti" de la Facultad de Florencia, en la que participaban varios de los futuros protagonistas radicales ${ }^{18}$.

Florencia se convierte así en el primer escenario de una progresiva politización ${ }^{19}$ que primero impregna el mundo universitario, en el que los estudiantes reclaman la renovación de un sistema educativo anclado en el conservadurismo de unas metodologías didácticas basadas en planteamientos neo-racionalistas ${ }^{20}$, y que más tarde guiará el desarrollo de la arquitectura experimental italiana hasta mediados de los 70 .

[11]

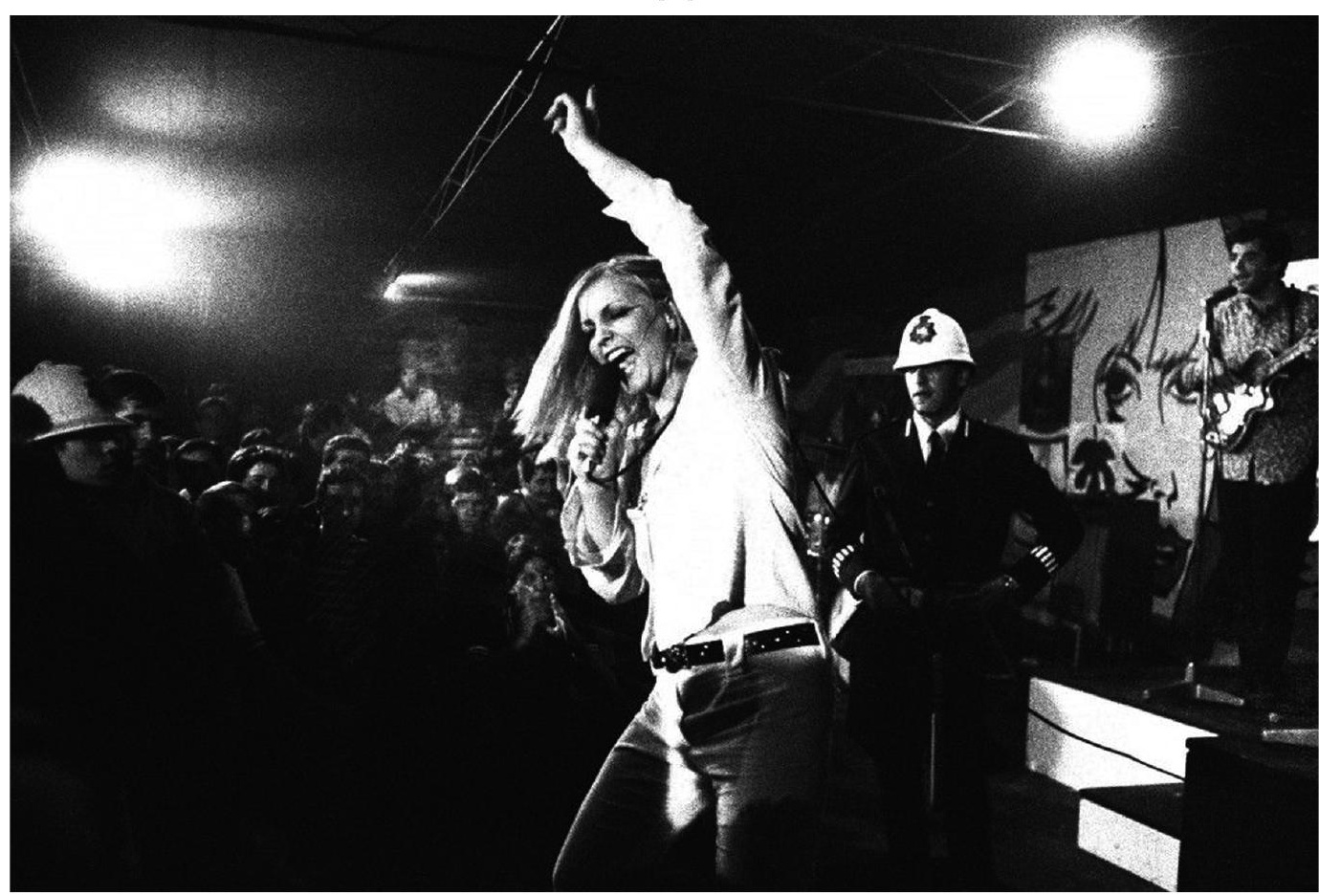


Inmersos en este ambiente reivindicativo, dos jóvenes profesores florentinos, Leonardo Savioli y Leonardo Ricci, convencidos del poder de la contaminación de pensamientos y la complementariedad de las artes, deciden desarrollar desde una perspectiva disidente nuevos métodos de trabajo aprovechando la Universidad como una ocasión óptima para fomentar la creación colectiva y abrir los campos de referencia a experiencias venidas del exterior. Progresivamente van integrando en sus cursos el estudio de las nuevas tecnologías asociadas a los medios de comunicación, el acercamiento a las megaestructuras como experimentos para la transformación utópica de la vida -influenciados por las noticias que llegaban de Londres- y las nuevas ideas de Eco sobre comunicación visual y el concepto de diseñador "semiológicamente consciente" ${ }^{21}$, imprimiendo también un nuevo sesgo político a sus enseñanzas para diferenciarse del "modelo oficial". Ambos se convertirán en inspiradores de una docena de arquitectos que algunos años después, conscientes de la crisis que atravesaba la arquitectura y ante la falta de expectativas profesionales ${ }^{22}$, deciden "vaciar" la disciplina ${ }^{23}$ para alcanzar un "grado cero" desde el que re-construir un universo formal nuevo ${ }^{24}$.

Precisamente Savioli, a través de su labor docente, es responsable en gran medida de la nueva percepción que esos jóvenes arquitectos y diseñadores italianos empiezan a tener acerca de los nuevos "palacios de la diversión", que ahora son observados como un laboratorio experimental estilístico y funcional capaz de generar modelos inspiradores de un nuevo orden social ligado al entretenimiento.

El detonante lo encontramos en el programa del nuevo curso académico de 1966-67 que Savioli organiza bajo el título "Spazio di coinvolgimento" [Espacio de participación] ${ }^{25}$ eligiendo el programa del Piper como ejercicio final, posiblemente influido por las primeras teorías situacionistas de la segunda mitad de los años 50 y su materialización más concreta -la enciclopédica "New Babylon" de Constant ${ }^{26}$ - , y por el debate surgido en Italia dos años antes con motivo de la celebración en 1964 de la XIII Triennale di Milano cuya "Sezione introduttiva a carattere internazionale", comisariada por Umberto Eco y Vittorio Gregotti, había sido dedicada al "Tempo libero"27 [12].

El objetivo del curso era abordar la necesaria implicación del arquitecto en el diseño de espacios que promovieran el comportamiento libre y la flexibilidad a través del movimiento, fomentando una nueva relación entre el usuario y su espacio, y a la vez rescatando zonas sometidas a la uniformidad de la rígida escenografía urbana. Términos como desorientación, transposición de escala, ensamblaje, montaje, flexibilidad, repetición, interacción, contaminación, son usados continuamente durante el curso gracias, en gran medida, al interés de Saviloli por el trabajo de Cedric Price ${ }^{28}$ en torno a su revolucionario proyecto del "Fun Palace" que, durante más de una
21 En esos mismos años (1965-69), Umberto Eco se convierte en profesor de "Comunicación visual" en la Facultad de Arquitectura de Florencia, donde defenderá la figura del diseñador "semiológicamente consciente" e invitará a los alumnos a pensar la arquitectura en términos mediáticos a través de la lectura astuta de las obras de McLuhan, que en 1967 serán analizadas en varios artículos publicados en la revista DOMUS

22 Entre 1965 y 1969 la industria de la construcción en Italia sufre una recesión importante, tanto en el sector público como en el privado.

23 "Queriamos vaciar la arquitectura de todo valor arquitectónico, es decir, convertirla en algo más. Queriamos vaciarla de su identidad disciplinaria y llenarla de vida". Paolo Deganello. Cita en MENKING, William; KAZI, Olimpia. Radical Italian Architecture Yesterday and Today. Architectural Design, Volume 77, Issue 3, Mayo/Junio 2007, pp. 99-101.

24 TORALDO DI FRANCIA, Cristiano. Memories of Superstudio. En LANG, Peter; MENKING, William. Superstudio: Life without objects. Milan: Skira, 2003, p. 68.

${ }^{25}$ SAVIOLI, Leonardo. Ipotesi de Spazio. Florencia, 1972.

${ }^{26}$ Para los situacionistas la sociedad futura que resultaria de la revolución estaria caracterizada por la conversión de todo tiempo en tiempo de ocio, puesto que la producción sería realizada por máquinas. Siguiendo al famoso sociólogo Johan Huizinga, el homo ludens sustituiria al homo faber. Ver Huizinga, J. Homo ludens (1938). Madrid, 1972. Uno de los primeros textos que Constant publica en el entorno situacionista es "El gran juego del futuro" (originalmente publicado en Potlatch 1, julio 1959) donde señala la necesidad de incluir lo lúdico en la vida social cotidiana y, por lo tanto, en el urbanismo.

27 Dentro de la Sección Internacional comisariada por Eco y Gregotti destaca la instalación del Caleidoscopio que el propio Gregotti diseña en colaboración con G. Stoppino, L. Meneguetti y P. Brivio. Se trataba de un monumental ambiente en forma de túnel de sección poligonal recubierto de
[12]
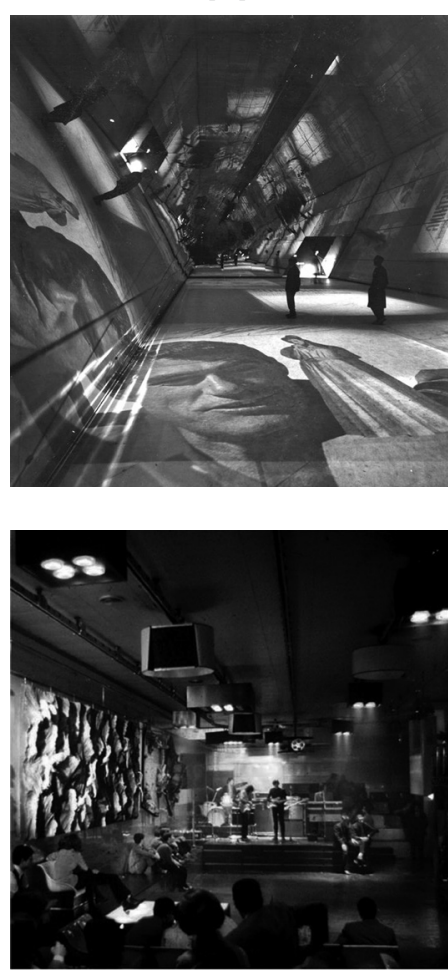

[14]
[15]

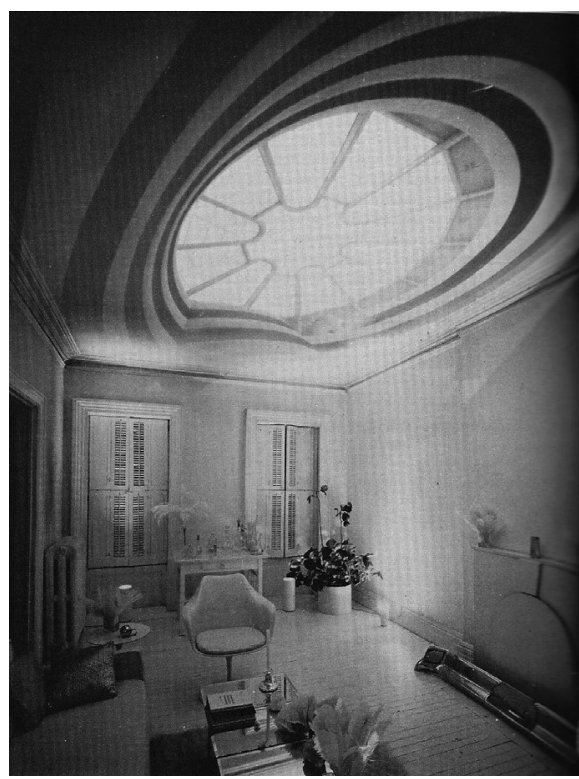

[16] Interior del L'Altro Mondo Club en Rimini (1967), espacio que fue definido por la revista Domus como "arquitectura eléctricamente ampliada". Fuente: www.laboratoireurbanismeinsurrectionnel. blogspot.com.es/2012/10/utopie-de-la-discotheque

[17] Interior del night-club Mach2 de Florencia (1968) diseñado por Superstudio y programado a partir de lineas de luz como únicos elementos de orientación. Fuente: www.cristianotoraldodifrancia.it/projects/interiors/66-73/67_mach2/67_mach2A
$[13$
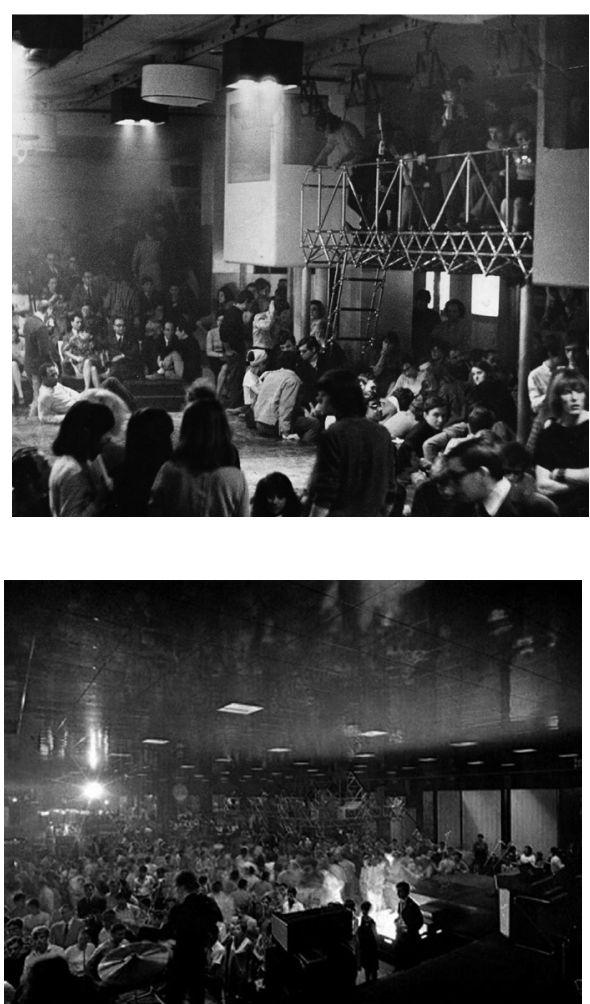

[16] 
espejos en los que se reflejaban las imágenes proyectadas sobre el suelo, acompañadas por música y diferentes sonidos. Para más información sobre las dos propuestas ver RINALDI, Marco. caleidoscopio: temi e stile dell'allestimento in Italia dal racionalismo alla neovanguardia. Roma: Bagatto Libri, 2003.

${ }^{\mathbf{2 8}}$ Ver MATHEWS, Stanley. From Agit-Prop to Free Space: The Architecture of Cedric Price. Londres: Black Dog Publishing, 2007.

${ }^{29}$ Ver LITTLEWOOD, Joan. Joan's Book: Joan Littlewood's Peculiar History as She Tells it. Londres: Methuen Publishing Ltd., 1994.

${ }^{30}$ MARCUSE, Herbert. Eros and Civilization A Philosophical Inquiry into Freud. Beacon Press, 1955.

31 SAVIOLI, Leonardo. Spazio di coinvolgimento. Casabella $326,1968, \mathrm{pp}$. $32-46$

32 CHIESA, Laura. Superstudio double-take: rescue operations in the realm of architecture. En CHIRUMBOLO, Paolo; MORONI, Mario; SOMIGLI, Luca. Neovanguardia. Italian Experimental Literature and Arts in the 1960s. University of Toronto Press, 2010 p. 287.

${ }^{33} \mathrm{~A}$ propósito del curso, en el que participa como asistente, Natalini escribe: "Spaesamento, trasposizione di escala, assemblaggio, montaggio, scomposizione, ripetizione, contaminazione, sono termini che sono stati continuamente usati, e soprattutto sono stati i nuovi stimoli che hanno dato alla progettazione lo scatto necessario a passare da materia di studo o da routine professionale ad azione creativa ed attiva" ["Desorientación, transposición de escala, ensamblaje, montaje, descomposición, repetición, contaminación, son términos que se utilizaban de forma continua y, sobre todo, se convirtieron en nuevos estimulos que imprimieron al proyecto el impulso necesario para pasar de la materia de estudio o de la rutina profesional a la acción creativa y activa"]. NATALINI, Adolfo. Arti visive e spazio di coinvolgimento. Casabella XXXII n³26, julio 1968, pp. 34-36. 34 NAVONE, Paola; ORLANDONI, Bruno. Architectura Radicale. Milán: Documenti di Casabella, 1974, p. 25.

${ }^{35}$ CALDINI, Carlo. Space Electronic. En COLES, Alex; ROSSI, Catharine. The Italian Avant-Garde, 1968-76. EP Vol.1. Berlin: Sternberg Press, 2013, p. 98

${ }^{36}$ La publicación en 1945 del libro de Bruno Zevi Verso un'architettura organica produce, a principios de los 60 , una ola de fascinación entre los arquitectos italianos por la obra de Frank Lloyd Wright, lo que hace que muchos estudiantes crucen el Atlántico obedientemente para conocerla de primera mano.

${ }^{37}$ La exposición coincide con la devastadora crecida del Arno de 1966, y parece lógico pensar que también utilicen esta catástrofe como episodio catártico.

38 "La superarchitectuttura e l'architettura della superproduzione, del superconsumo, della superinduzione al consumo, del supermarket, del superman e della benzina super. La superarchitettura accetta la logica della produczione e del consumo e vi esercita un'azione demistificante." ["La Superarquitectura es la arquitectura de la superproducción, del superconsumo, de la superinducción al consumo, del supermercado, del superhombre, de la gasolina súper. La Superarquitectura acepta la lógica de la producción y del

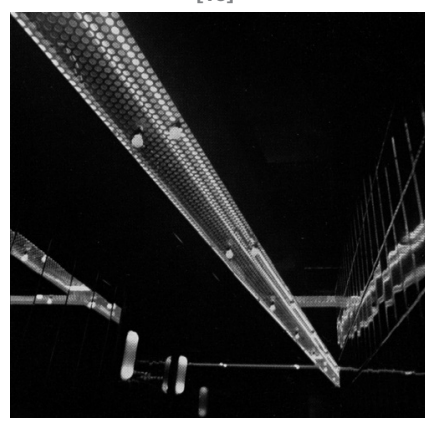

década y bajo el impulso de la promotora teatral Joan Littlewood -considerada la "madre" del teatro moderno y fundadora del Theatre Workshop ${ }^{29}-$, quiso convertirse en el gran "laboratorio de la diversión" resultado de la fusión entre ocio, aprendizaje y creación en un entorno en continua transformación auto-gestionado por los propios usuarios. Price, en colaboración con el cibernético Gordon Pask, es el primero en "soñar" con un edificio completamente re-programable capaz de adaptarse a un uso (futuro) impredecible con la ayuda de sistemas informáticos interactivos diseñados para aprender e improvisar junto a los humanos. Como veremos, sus investigaciones en torno al "Fun Palace", considerado la representación del impulso lúdico a la creación que Marcuse había colocado en la base de su crítica a la sociedad totalitaria en su Eros y Civilización ${ }^{30}$, resuenan constantemente en la producción posterior vinculada a las neo-vanguardias, y abordan desde otra perspectiva muchas de las preocupaciones y demandas de sus protagonistas.

El curso queda documentado en un artículo publicado en Casabella en $1968^{31}$ donde, además de constatar las evidentes influencias procedentes del mundo de la arquitectura, también se explica, tal y como apunta Laura Chiesa, cómo el proyecto trataba de incorporar a la propia arquitectura dos experiencias artísticas contemporáneas: el Pop Art (con su adhesión al "mundo de la imagen" y a la sociedad del consumo) y el Arte Programmata (con sus reflexiones sobre la reiteración, repetibilidad y seriación) ${ }^{32}$. Varios de los participantes, bien como asistentes o como alumnos, se convertirán en los futuros protagonistas del fenómeno radical, entre ellos Adolfo Natalini ${ }^{33}$ (fundador de Superstudio), Paolo Deganello (cofundador de Archizoom), Pietro Derossi, Dario Bartolini (futuro integrante de Archizoom), Alessandro Poli y Alessandro Magris (futuros integrantes de Superstudio), Carlo Bachi (cofundador de UFO), Alberto Breschi y Giuliano Fiorenzuoli (cofundadores del grupo Zziggurat) o Paolo Galli y Fabrizio Fiumi (fundadores del grupo 9999) ${ }^{34}$.

Este último, todavía como estudiante y en compañía de su futuro socio en 9999, Carlo Caldini ${ }^{35}$, visita E.E.U.U. por segunda vez en 1967 y cambia la experiencia helicoidal del Guggenheim de su primer viaje $^{36}$ por la inmersión nocturna en una de las sesiones multi-sensoriales orquestadas por Warhol en el Electric Circus. El sorprendente cambio de intereses, más allá de los evidentes y asumidos impulsos juveniles, está motivado, primero, por la influencia que sobre él ejerce el profesor Savioli a través de aquellos cursos en los que cuestionaba el "modelo oficial" de enseñanza mediante la integración del estudio de las nuevas tecnologías asociadas a los medios de comunicación y el acercamiento a experiencias venidas del exterior $y$, segundo, por el doble estímulo que supone la irrupción en 1966 de dos proyectos protagonizados por varios compañeros de universidad: la exposición "Superarchitettura" y la inauguración en Turín del Piper Pluriclub.

La primera es escenificada en dos actos -en las ciudades de Pistoia y Módena- por cinco antiguos alumnos de la Facultad de Florencia recién titulados (Andrea Branzi, Gilberto Corretti, Paolo Deganello, Massimo Morozzi y Adolfo Natalini) y constituye un punto de inflexión en la vida de estos jóvenes arquitectos que, inmersos en un ambiente de profunda frustración ${ }^{37}$ y movidos por una inquebrantable actitud crítica, deciden desmitificar la nueva sociedad altamente orientada hacia los medios y el consumo a través de la celebración irónica de la abundancia ${ }^{38}$, celebrando

$[19]$

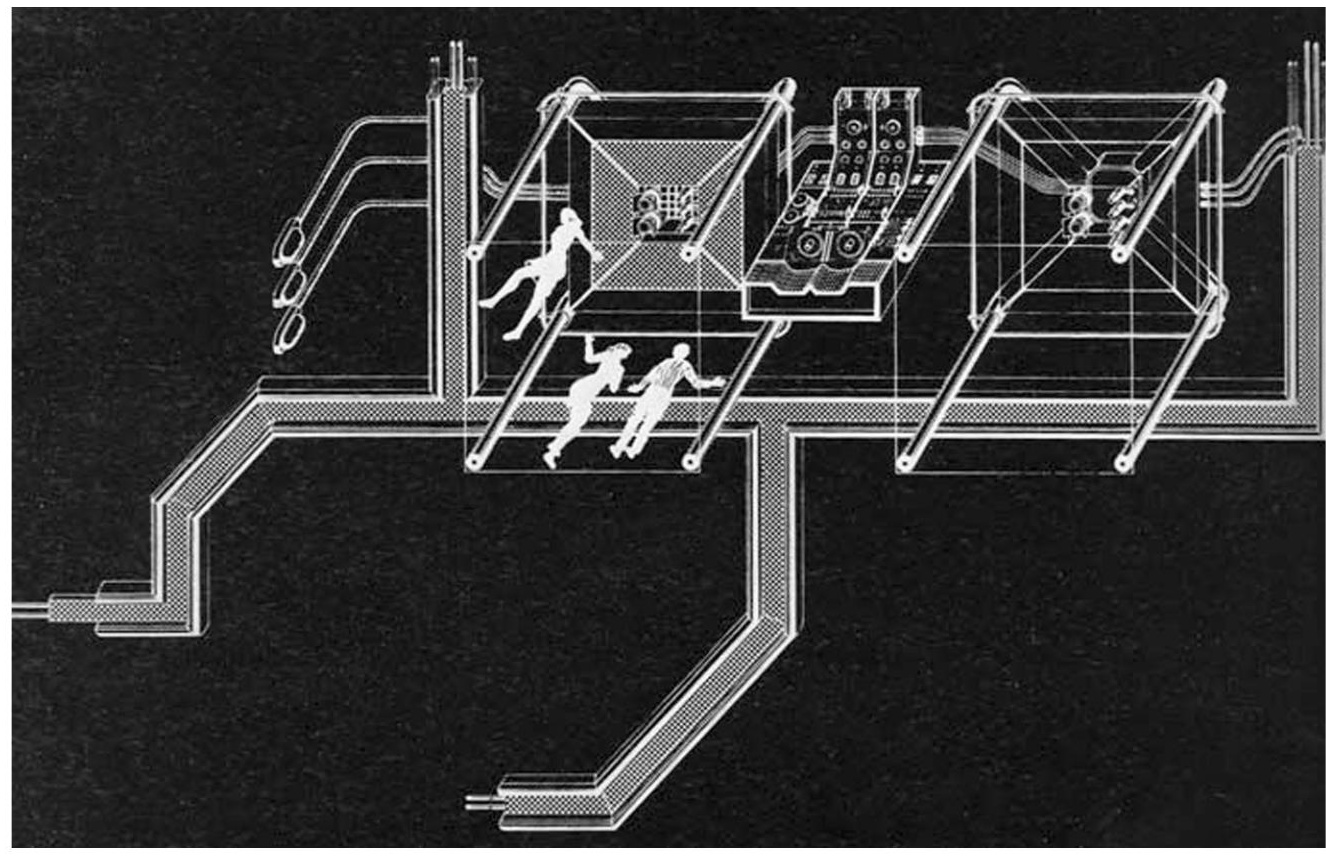




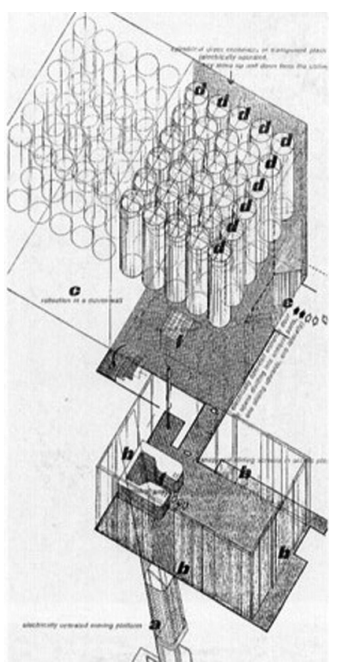

[20]

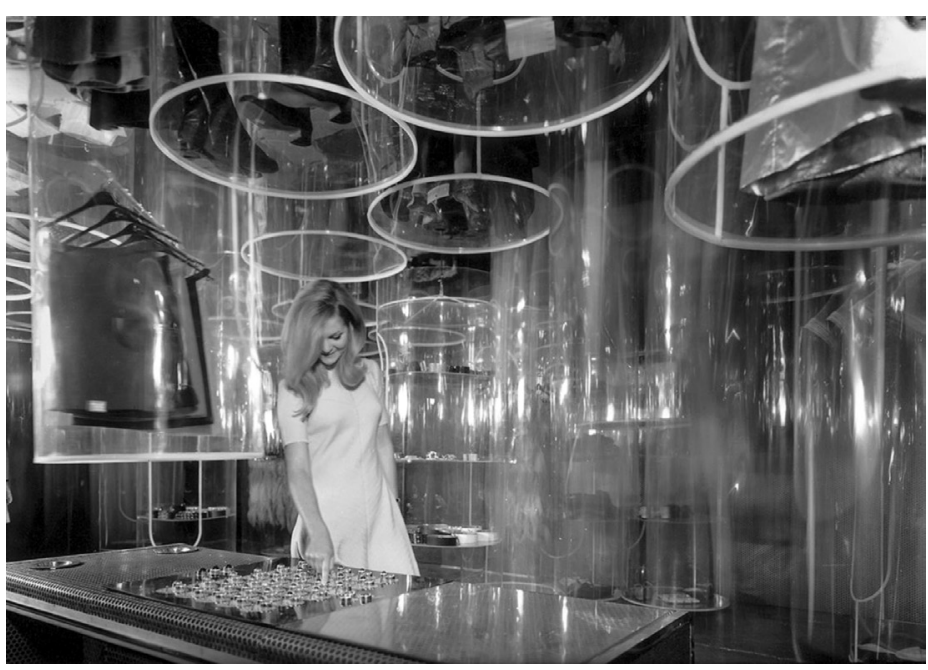

[21]

lo que muchos apuntan como el evento fundacional del breve periodo radical italiano. La segunda exposición también servirá como acto fundacional de dos de los grupos más influyentes de este periodo, Archizoom y Superstudio ${ }^{39}$.

Al mismo tiempo en 1966 abre sus puertas el Piper Pluriclub, espacio multifuncional diseñado y gestionado por el joven Pietro Derossi que inmediatamente revoluciona la escena "alternativa" turinesa al ser concebido como una máquina subversiva equipada con los "effetti luminosi" diseñados por Bruno Munari como decoración variable y móvil que condensaba sus experiencias previas en torno a las "pinturas de luz"40. Tanto el espacio como el equipamiento audiovisual del local estaban pensados para activar la implicación instantánea del público a través de una "programación" [13 y 14] que le permitía albergar todo tipo de actividades: desde el baile para los trabajadores de la Fiat los viernes por la noche, las representaciones del Living Theater -ya inmerso en su legendaria migración europea de más de cuatro años ${ }^{41}$ - previstas para la tarde de los miércoles, hasta la adaptación como sala de exposiciones-espacio cultural durante el resto del día ${ }^{42}$.

No sorprende, por tanto, que en esta segunda ocasión Fiumi y Caldini desembarquen en NY en busca de nuevas sensaciones más vinculadas al ocio y al control de entornos ideales que a la propia arquitectura entendida como el acto de construir supeditado a un resultado formal fijo.

Tampoco sorprende que de vuelta en Florencia se unan al hiperactivo grupo de jóvenes -entre los que ya destacaban las experiencias milanesas de Ugo la Pietra en torno a los "grados de libertad", o las investigaciones de Riccardo Dalisi, que desde los barrios humildes de Nápoles anticipaban la posterior "arquitectura participativa"- que, estimulados por la tecnología y el invasivo mundo pop y cargados de compromiso social y político, trasladan intencionadamente su interés desde la forma construida a la generación de ambientes artificiales con objetivos ideológicos, al considerar estos lugares electrónicamente amplificados como la afirmación de una nueva emancipación social ${ }^{43}$ (el espectador se convierte en actor) y como los únicos capaces de expresar el concepto

consumo, operando en ella con acciones de desmitificación."] Manifesto de la $2^{\mathrm{a}}$ exposición Superarchitettura, Sala Comunale de Modena, 1967.

${ }^{39}$ Andrea Branzi, Gilberto Corretti, Paolo Deganello, Massimo Morozzi y posteriormente los diseñadores Dario y Lucia Bartolini forman Archizoom, mientras que Adolfo Natalini, al que se une tras la exposición Cristiano Toraldo di Francia, forman Superstudio, grupo al que posteriormente se unen gradualmente Roberto Magris, Gian Piero Frassinelli, Alessandro Magris y Alessandro Poli.

40 Domus $n^{\circ} 446$. Enero 1967.

41 El polémico The Living Theater, primer grupo de teatro experimental estadounidense, fundado en 1947, tras varios intentos de resistencia, más denuncias y algunas semanas en prisiones federales, finaliza su primera aventura norteamericana en 1964, año en el que comienza su ya legendaria migración de más de cuatro años a lo largo de Europa, durante la cual crean sus espectáculos más importantes. El Living europeo no solo realiza sus mejores puestas en escena y

desarrolla muchos conceptos y metodologías fundamentales como la creación colectiva, la improvisación como herramienta de creación y de representación o la inclusión del público en los espectáculos, sino que también construye una comunidad de convivencia y trabajo organizada a partir de unos ideales anarco-pacifistas que intentan promover en la sociedad. Una de sus "paradas" la realizan en el Piper Pluriclub de Turín.

42 Las conexiones de Derossi con el incipiente Arte Povera convirtieron el Piper Pluriclub de Turin en punto de encuentro para artistas y exposiciones ligadas a este movimiento.

43 En este sentido, y en sintonía con el "espiritu de los tiempos", Andrea Branzi defendía que el impulso de "actividades sicomotrices como el baile o el sexo entendidas como la base de la comunicación espontánea, creaba una nueva libertad" y "consecuentemente un nuevo impulso político dirigido a la destrucción de un equilibrio ficticio basado en la inhibición". BRANZI, Andrea. Radical Notes. Rock e Rivoluzione. Casabella 374, 1973, p.10.

44 "The spatial model of the Pipers consisted in a sort of immersion in a continuous flow of images, stroboscopic lights and very loud stereophonic music; the goal was total estrangement of the subject, who gradually lost control of his inhibitions in dance, moving towards a sort of psychomotor liberation. This did not mean for us a passive surrender to the consumption of aural and visual stimuli, but a liberation of the full creative potential of the individual. In this sense the political significance of the Pipers is evident as well." ["El modelo espacial de los Pipers consistía en una especie de inmersión en un flujo continuo de imágenes, luces estroboscópicas y música estereofónica muy alta; el objetivo era el
[18] Perspectiva interior del night-club Mach2 de Superstudio. CSuperstudio, 1967. Fuente: revista Domus n473, Abril 1969. Pág.29.

$[19,20]$ Perspectiva e interior del Bang Bang Club unido a la tienda Altre Cose, diseñados por Ugo la Pietra en Milán (1968). CUgo la Pietra, 1968. Fuente: www.ugolapietra.com/Negozio_Altre_Cose.htm?c=arch\&m=1 $Y$ www.tectonicablog.com/?p=85529

[21] Interior del Space Electronic de Florencia (1969). Fuente: www.spaceelectronic.wordpress. com/2014/06/04/archival-photographs/\#jp-carousel-72

[22] Performance del Living Theater en el Space Electronic (1969). Fuente: www.spaceelectronic.wordpress. com/2014/06/04/archival-photographs/\#jp-carousel-80

[23] "Vegetable garden", instalación del Gruppo 9999 durante la celebración del Mondial Festival en 1971 incluida dentro de las actividades del proyecto S-Space (School for Expanded Conceptual Architecture) fundado por el Gruppo 9999 y Superstudio en 1970 en Florencia, con la discoteca Space Electronic como base de operaciones. (C) Gruppo 9999. Carlo Caldini, 1971.
[22]

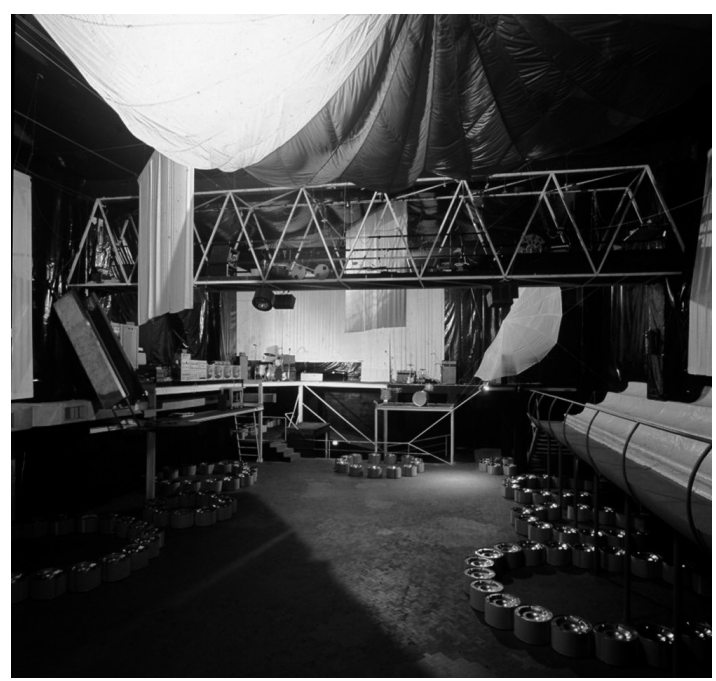


distanciamiento total del sujeto, el cual pierde gradualmente el control de sus inhibiciones a través del baile, desplazándose hacia una especie de liberación psicomotora. Esto no significaba para nosotros una rendición pasiv al consumo de estimulos auditivos y visuales, sino una liberación de todo el potencial creativo del individuo. En este sentido, el significado politico de los Pipers es también evidente."] BRANZI, Andrea. La casa calda: Esperienze del nuovo design italiano. Idea Books, 1984, p. 54.

45 "Breve storia dello stile YÉYÉ". RESTANY, Pierre. Domus 446 (1967).

${ }^{46}$ Consultar el maravilloso libro de SMITH, C Ray. Supermannerism. New Attitudes in Post Modern Architecture. New York: E. P. Dutton, 1977, p. 298.

47 COOK, Peter. Experimental Architecture. New York: Universe Books, 1970, pp. 83-84, 141.

48 "Designing for delight and pleasure should very seldom be seen to happen, and must encompass-indeed nurture-doubt, danger, mystery and magic... Distortion of time, space and substance is as necessary a design tool for pleasure as it is for religious architecture." ["Diseñar por disfrute y placer resulta raro de ver, y debería abarcar -incluso nutrirse de- la duda, el peligro, el misterio y lo mágico... La distorsión del tiempo, el espacio y la sustancia es una herramienta de diseño tan necesaria para el placer como lo es para la arquitectura religiosa."] PRICE, Cedric. Life- Conditioning Architectural Design 36, 1966.

49 En 1960 Pierre Restany, apercibiéndose del atractivo promocional derivado de la organización de artistas en un colectivo que operase bajo una misma bandera, organiza un grupo de ellos en París con la intención de crear un movimiento de vanguardia y forma el Nouveau Réalisme (vertiente europea del Pop americano según Peter Bürger). El manifiesto fundacional fue firmado el 27 de octubre en e apartamento de Yves Klein por el mismo Klein y Restany, Arman, Francois Dufrêne, Raymond Hains, Martial Raysse,Daniel Spoerri, Jean

Tinguely y Jaques de la Villeglé, para reclamar un nuevo enfoque perceptivo de lo real

("Nuevo Realismo=nuevas percepciones de lo real").

${ }^{50}$ CALDINI, Carlo. Space Electronic. En COLES, Alex; ROSSI, Catharine. The Italian Avant-Garde, 1968-76. EP Vol.1. Berlin: Sternberg Press, 2013, pp 97-105.

51 "El fin último de la arquitectura moderna es la eliminación de la propia arquitectura". ARCHIZOOM en la introducción de NAVONE, Paola; ORLANDONI, Bruno. Architectura Radicale. Milán: Documenti di Casabella, 1974.

52 CALDINI, Carlo. Ricordi di Architettura. Auto-publicación del Gruppo 9999. Florencia 1972. de modernidad al aprovechar su elasticidad atmosférica ${ }^{44}$ para incorporar el compromiso con las formas y la lógica de las nuevas tecnologías y los medios de comunicación.

Ya en 1967 el crítico Pierre Restany anunciaba en las páginas de Domus la aparición de "un nuevo tipo de ambiente, que no es solo un club nocturno, sino un lugar de reuniones, exposiciones y otros eventos. Es un nuevo tipo de espacio, continuamente roto y modificado por efectos de luz, un espacio ilusorio creado a partir de dispositivos, proyectores, reflectores, así como la música es creada por instrumentos: un espacio que no existe sino solo cuando está en acción"45. El precursor del Nouveau Réalisme definía así aquellos primeros experimentos de interiores instantáneos -surgidos paralelamente a las experiencias de C. Ray Smith en torno a la manipulación de ambientes domésticos utilizando dispositivos de proyección ${ }^{46}$ [15]- que respondian automáticamente a estilos y tendencias y que, según Peter Cook, confirmaban evidentemente- las ideas de Cedric Price ${ }^{47}$ en torno a la adaptabilidad extrema como respuesta a un uso impredecible y al aprovechamiento de las nuevas tecnologías como instrumento de reprogramación ${ }^{48}$.

El resultado inmediato fue la aparición de varios proyectos en los que se confirmaba de alguna manera aquel "nuevo enfoque perceptivo de lo real" reclamado unos años antes por el propio Restany ${ }^{49}$ y escenificado en locales como el "L'Altro Mondo Club" de Pietro Derossi en Rimini (1967) [16], el "Mach 2" de Superstudio en Florencia (1967) [17 y 18], el Bang Bang unido a la tienda Altre Cose de Ugo la Pietra en Milán (1968) [19 y 20], o el mítico y todavía activo "Space Electronic" del Gruppo 9999 (Fiumi, Cladini \& Co.) en Florencia (1969) ${ }^{50}$ [21,22 y 23], ejemplos de arquitectura como catalizadora de una pulsión social que mezclaba en el mismo espacio la vanguardia cultural y experimental más radical con el fenómeno de masas de la sociedad del espectáculo. Para estos jóvenes arquitectos italianos, además, diseñar un Piper significaba explotar la capacidad de los nuevos espacios de participación para liberar las fuerzas de aquella guerra existencial en la que se encontraban inmersos y con la que pretendían romper los límites de una arquitectura heredada incapaz de actuar contra los poderes establecidos.

Italia se convierte así en el marco perfecto para la expansión del denominado "fenómeno Piper" que, planificado durante una larga noche de hace casi 50 años, articula y explota el encuentro (premeditado) entre Warhol-McLuhan-Price en torno al potencial ideológico representado por el espacio del night-club, un potencial descubierto por una nueva generación de arquitectos y artistas que, como usuarios directos, ahora aplicaban en forma de liberación corporal y sicológica vinculada sin complejos a la música popular y a los excesos consumistas, algo que consideraban imposible de alcanzar dentro de las rígidas estructuras de aquella arquitectura heredada que pretendían superar ${ }^{51}$.

Finalmente, las propuestas asociadas a este fenómeno serán etiquetadas para describir la celebración instantánea de una arquitectura "eléctrica" entendida como reflejo operativo del "Palacio de la diversión" y pensada para absorber la complejidad de la mediatizada sociedad contemporánea como excusa para expandir ilimitadamente su campo de operaciones.

Podemos decir entonces que sí..."se puede diseñar con electricidad"52.
[23]

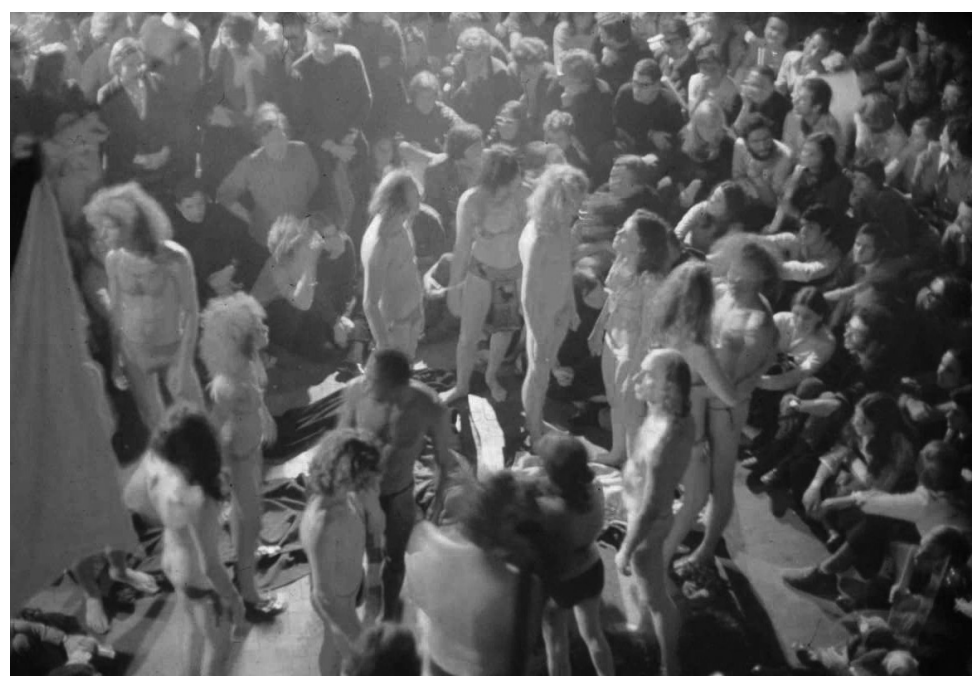

[24]

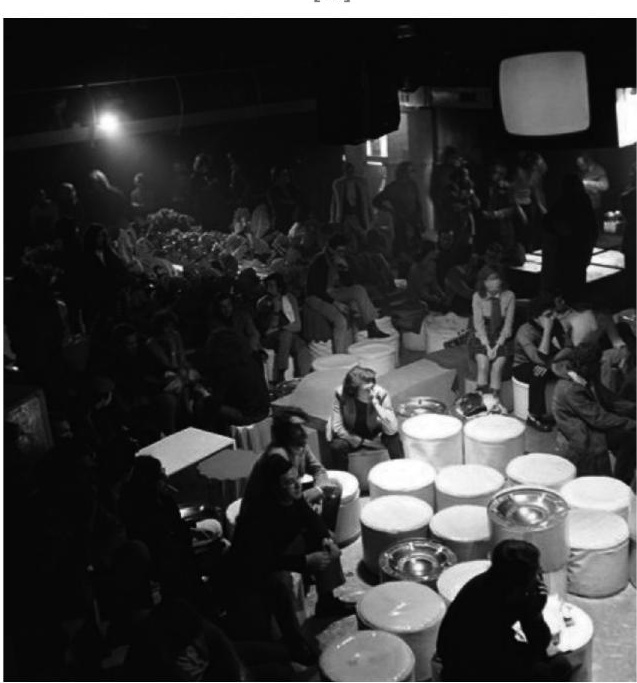

